\title{
Вольт-амперные характеристики диодов Шоттки при больших плотностях тока в условиях инжекции неосновных носителей
}

\author{
(C) T.T. Мнацаканов ${ }^{1}$, А.Г. Тандоев ${ }^{1}$, M.E. Левинштейн ${ }^{2}$, C.H. Юрков ${ }^{1}$, J.W. Palmour ${ }^{3}$ \\ ${ }^{1}$ Всероссийский электротехнический институт им. В.И. Ленина, \\ 111250 Москва, Россия \\ ${ }^{2}$ Физико-технический институт им. А.Ф. Иоффе Российской академии наук, \\ 194021 Санкт-Петербург, Россия \\ ${ }^{3}$ Wolfspeed, A Cree Company, 3026 East Cornwallis Rd., Research Triangle Park, \\ NC 27709, USA \\ E-mail: mnatt@yandex.ru
}

(Получена 30 января 2017 г. Принята к печати 15 февраля 2017 г.)

\begin{abstract}
Получено аналитическое выражение для вольт-амперной характеристики диода Шоттки при высоком уровне инжекции неосновных носителей заряда. Вплоть до самых высоких значений плотности тока падение напряжения на диоде тем больше, чем выше уровень легирования базы. Проанализирован физический механизм, обусловливающий возникновение этого „парадоксального“ результата. Численный расчет с помощью программы, учитывающей полную совокупность нелинейных эффектов, обусловленных высоким уровнем инжекции в базовом слое и сильным легированием эмиттерной области, подтверждает справедливость полученного аналитического результата.
\end{abstract}

DOI: 10.21883/FTP.2017.08.44801.8478

\section{1. Введение}

Диоды Шоттки (ДШ) в настоящее время являются одними из самых распространенных приборов современной полупроводниковой электроники. Мощные ДШ широко применяются при создании устройств компенсации реактивной мощности, коммутаторов солнечных батарей, в автомобильной электронике. Преимуществами ДШ перед приборами с $p$-n-переходом являются быстродействие, малые прямые падения напряжения и малые коммутационные потери. Эти преимущества в основном обусловлены тем, что в стандартных рабочих режимах при протекании прямого тока отсутствует накопление неосновных носителей в базе ДШ. Однако в режимах короткого замыкания и ударных токовых нагрузок прямые токи могут в десятки раз превосходить токи, характерные для рабочих режимов (см., например, [1-3]).

Уже в первых работах, посвященных исследованию ДШ, отмечалось, что при достаточно больших прямых смещениях может наблюдаться инжекция неосновных носителей из контакта металл-полупроводник. Более того, при достаточно больших плотностях прямого тока в базе ДШ может реализовываться режим высокого уровня инжекции [4-6]. Наиболее подробное исследование процессов, возникающих в диодах Шоттки при высоких уровнях инжекции, проведено в рамках классических представлений в работе [7].

В последнее время интерес к поведению диодов Шоттки в присутствии неосновных носителей заряда вновь возник в связи с появлением и быстрым развитием ДШ на основе карбида кремния. В обычных условиях неосновные носители возникают в базе ДШ, если высота барьера Шоттки превышает половину ширины запрещенной зоны $E_{g}$. В связи с большой величиной $E_{g}$ в $\mathrm{SiC}$ казалось, что проблемы, связанные с появлением неосновных носителей заряда для ДШ на основе $\mathrm{SiC}$, неактуальны. Тем не менее появление неосновных носителей в базе $4 H$-SiC ДШ отмечалось в работе [8].

Однако в основном интерес к поведению неосновных носителей заряда в карбидкремниевых структурах обусловлен широким распространением JBS-приборов (junction barrier Schottky diodes), представляющих собой интегрированные структуры, в которых области с диодами Шоттки перемежаются с локальными $p-n$-областями [9]. В настоящее время практически все мощные и высоковольтные структуры ДШ представлены именно JBS-приборами [10].

Протекание прямого тока через JBS-структуру в первом приближении может быть описано как параллельное соединение ДШ и $p$-n-перехода [11]. При больших плотностях тока вольт-амперные характеристики как ДШ, так и $p-n$-перехода должны быть рассчитаны с учетом эффектов, выявленных и описанных недавно для $p-n$-переходов $[12,13]$. При больших значениях плотности прямого тока наряду с хорошо известными диффузионным и дрейфовым режимами необходимо учитывать существование режима диффузии, стимулированной квазинейтральным дрейфом (DSQD mode) [12]. Характеристики этого режима отличаются важными особенностями и существенно влияют на вид прямой вольт-амперной характеристики. Кроме того, при больших плотностях тока необходим учет полевых зависимостей подвижностей электронов и дырок, $\mu_{n}(F)$ и $\mu_{p}(F)$, даже если удовлетворяется условие квазинейтральности [13].

В работе [14] с учетом этих эффектов вольт-амперная характеристика ДШ была аналитически рассчитана для высокого уровня инжекции неосновных носителей при 
относительно небольших плотностях тока, соответствующих условию $j<j_{c r n}$, где

$$
j_{c r n}=q\left(\mu_{n 0}+\mu_{p 0}\right) N_{d}\left(F_{s n}^{-1}-F_{s p}^{-1}\right)^{-1} .
$$

Здесь $q-$ элементарный заряд, $\mu_{n 0}$ и $\mu_{p 0}-$ подвижности в слабом электрическом поле электронов и дырок соответственно, $N_{d}-$ уровень легирования $n$-базы диода Шоттки, $F_{s n}$ и $F_{s p}$ - характерные значения электрического поля, при котором дрейфовые скорости электронов и дырок соответственно достигают насыщения. При этом в работе [14] был получен кажущийся парадоксальным результат: чем выше уровень легирования базы ДШ, тем выше падение напряжения на диоде при той же плотности тока.

При $N_{d}=10^{14} \mathrm{~cm}^{-3}$ значения $j_{c r n}$ составляют $\sim 340$ и $\sim 460 \mathrm{~A} / \mathrm{cm}^{2}$ для кремния и карбида кремния соответственно. Вместе с тем в режимах ударных токов и токов короткого замыкания плотности тока в $\mathrm{SiC}$-приборах существенно превышают приведенные выше значения. Так, в режиме так называемых ,длинных“ импульсов ударного тока с характерной длительностью, составляющей половину периода промышленной частоты 50 (или 60) Гц, значения плотности тока могут составлять $\sim(6000-9000) \mathrm{A} / \mathrm{cm}^{2}[1,14]$. В режиме „коротких“ импульсов со стандартной длительностью $8 / 20$ мкс, т.е. треугольных импульсов с временем нарастания 8 мкс и временем спада 20 мкс, значения плотностей ударных токов могут составлять $\sim(60-100) \mathrm{\kappa A} / \mathrm{cm}^{2}[15]$.

В настоящей работе аналитически исследуется вид вольт-амперных характеристик ДШ при высоком уровне инжекции неосновных носителей в области плотностей токов, отвечающих условию $j>j_{c r n}$. Аналитические результаты подкреплены численным моделированием.

\section{2. Постановка задачи}

Как и в работе [14], рассмотрим для определенности ДШ с базой $n$-типа проводимости, равновесная зонная

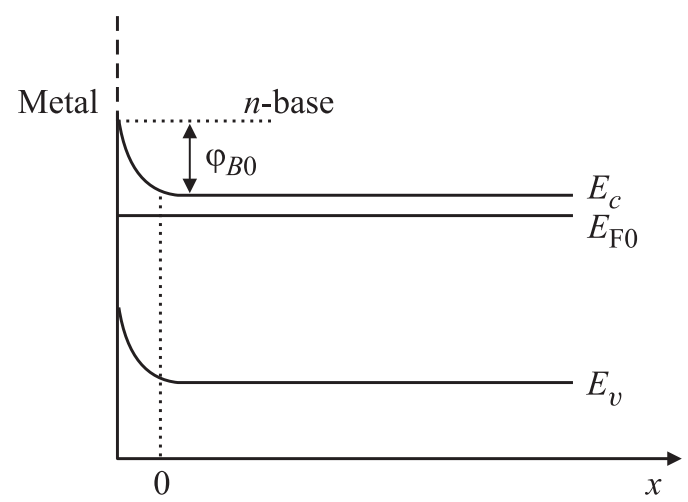

Рис. 1. Равновесная зонная диаграмма диода Шоттки с базой $n$-типа проводимости. Пунктиром показана граница области объемного заряда в $n$-базе. $E_{c}-$ край зоны проводимости, $E_{\mathrm{F} 0}-$ уровень Ферми. диаграмма которого показана на рис. 1. Для расчета вольт-амперной характеристики ДШ воспользуемся моделью, предложенной в работе [14]. В рамках этой модели уравнение непрерывности при высоком уровне инжекции носителей заряда в базовом $n$-слое может быть записано в виде

$$
\frac{d^{2} p}{d x^{2}}+\frac{j}{j_{d}}\left(\frac{j}{j_{c r}}-1\right) \frac{N_{d}^{2}}{p^{2} L} \frac{d p}{d x}=\frac{p}{L^{2}}
$$

где $j_{d}=2 q D_{p}(b+1) N_{d} / L, L=\left\{[2 b /(b+1)] D_{p} \tau\right\}^{1 / 2}-$ амбиполярная диффузионная длина, $b=\mu_{n 0} / \mu_{p 0}, D_{p}-$ коэффициент диффузии дырок, $\tau-$ время жизни неравновесных носителей в базе диода.

Граничное условие к уравнению (2), согласно результатам работы [14], имеет вид

$$
\left.\frac{d p}{d x}\right|_{x=0}=-\frac{j}{2 q D_{p}}+\frac{j_{s 0}}{q D_{a}} \frac{p^{2}(0)}{n_{i}^{2}}+\frac{j}{j_{d}}\left(\frac{j}{j_{c r}}-1\right) \frac{N_{d}^{2}}{p(0) L},
$$

где $j_{s 0}=A^{*} T^{2} \exp \left(-q \varphi_{B 0} / k T\right)-$ плотность тока насыщения перехода металл-полупроводник, $A^{*}-$ эффективная постоянная Ричардсона [14].

Граничное условие (3) отличается от „классического“ граничного условия, обычно используемого для анализа работы диода Шоттки при высоком уровне инжекции [16], наличием третьего члена в правой части. Как показано в работе [14], при формулировании граничного условия в работе [16] не была принята во внимание слабая инжекционная способность перехода метал-полупроводник (большая величина $j_{s 0}$ ). Это обстоятельство приводит к уменьшению концентрации неосновных носителей вблизи перехода и, следовательно, к возрастанию в этой области электрического поля. В таких условиях необходимо учитывать перенос носителей заряда в режиме диффузии, стимулированной квазинейтральным дрейфом (DSQD), подробно описанный и проанализированный в работах $[12,13]$. Как показано в работе [14] и будет продемонстрировано далее, использование граничного условия в форме, установленной в работе [16], т.е. пренебрежение третьим членом в равенстве (2), ведет к качественно неверным результатам.

Граничное условие на правой границе базы в точке $x=W(W-$ ширина $n$-базы) имеет обычный вид [14]:

$$
\left.\frac{d p}{d x}\right|_{x=W}=\frac{j}{2 q D_{n}}-\frac{j_{s p}}{q D_{a}} \frac{p^{2}(W)}{n_{i}^{2}} .
$$

Здесь $j_{s p}-$ плотность тока насыщения $n^{+}-n$-перехода на правой границе базы в точке $x=W$.

\section{3. Результаты и обсуждение}

Нелинейность уравнения (2) затрудняет его аналитическое решение. Поэтому, как и в работе [14], мы воспользуемся методом региональных приближений [16]. 
Поскольку, как указывалось выше, плотность тока насыщения (величина $j_{s 0}$ ) превосходит значение $j_{s p}$ на несколько, $\sim(4-5)$, порядков, концентрация носителей заряда на границе металл-полупроводник, $p(0)$, оказывается значительно меньше, чем $p(W)$. Поэтому с учетом типичного для ДШ соотношения $W / L<1$ концентрация инжектированных дырок $p(x)$ монотонно возрастает от $p(0)=p_{0}$ до $p(W)=p_{W}$.

В области базового слоя, примыкающей к контакту металл-полупроводник, $0 \leq x \leq x_{1}$, членом $p / L^{2}$ в уравнении непрерывности (2), описывающем рекомбинацию носителей заряда, вследствие малой концентрации неосновных носителей можно пренебречь. При длине этой области $x_{1}$ в области $0 \leq x \leq x_{1}$ уравнение непрерывности приобретает вид

$$
\frac{d^{2} p}{d x^{2}}+\frac{j}{j_{d}}\left(\frac{j}{j_{c r}}-1\right) \frac{N_{d}^{2}}{p^{2} L} \frac{d p}{d x}=0 .
$$

В отличие от соответствующего уравнения непрерывности для случая $j<j_{c r}[14]$ дрейфовый член в уравнении (5) положителен. Соответственно диффузионный член $d^{2} p / d x^{2}$ в уравнении (5) отрицателен. (В случае $j<j_{c r}$ диффузионный член во всей базовой области положителен [14]).

В области $n$-базы, примыкающей к $n-n^{+}$-переходу, $x_{2} \leq x \leq W$, высокая инжектирующая способность $n-n^{+}$-перехода $\left(j_{s p} \ll j_{s 0}\right)$ обеспечивает большую концентрацию носителей и обусловливает пренебрежимо малую величину дрейфового слагаемого по сравнению с диффузионным и рекомбинационным членами. В результате уравнение непрерывности в этой области имеет вид

$$
\frac{d^{2} p}{d x^{2}}=\frac{p}{L^{2}}
$$

Положения точек $x=x_{1}$ и $x=x_{2}$ определяются очевидными условиями

$$
\begin{aligned}
& \frac{p_{1}}{L^{2}}=\left.\frac{j}{j_{d}}\left(\frac{j}{j_{c r}}-1\right) \frac{N_{d}^{2}}{p_{1}^{2} L} \frac{d p}{d x}\right|_{x=x_{1}}, \\
& \frac{p_{2}}{L^{2}}=\left.\frac{j}{j_{d}}\left(\frac{j}{j_{c r}}-1\right) \frac{N_{d}^{2}}{p_{2}^{2} L} \frac{d p}{d x}\right|_{x=x_{2}},
\end{aligned}
$$

где $p_{1}=p\left(x_{1}\right), p_{2}=p\left(x_{2}\right)$.

В области $0 \leq x \leq x_{1}$ решение уравнения непрерывности (5) с граничными условиями (3) и (7) имеет вид

$$
\frac{p^{*}}{C L}\left\{\frac{p(x)-p_{0}}{p^{*}}-\frac{p^{*}}{C L} \ln \left[\frac{p(x) / p^{*}+p^{*} / C L}{p(0) / p^{*}+p^{*} / C L}\right]\right\}=\frac{x}{L},
$$

где

$$
\begin{gathered}
p^{*}=N_{d} \sqrt{\frac{j}{j_{d}}\left(\frac{j}{j_{c r}}-1\right)} \\
p(0)=p_{0}=n_{i} \sqrt{\frac{b}{b+1} \frac{j}{j_{s}}\left(1+\frac{b+1}{b} \frac{1 q D_{a} p_{W}}{j W}\right)} \\
C=\frac{p_{1}}{L}\left(\frac{p_{1}}{p^{*}}\right)^{2} .
\end{gathered}
$$

Протяженность области $0 \leq x \leq x_{1}$ может быть определена, если в решении (9) уравнения непрерывности (5) положить $p=p_{1}$. После несложных преобразований величину $x_{1} / L$ можно представить в виде

$$
\frac{x_{1}}{L}=\left(\frac{p^{*}}{p_{1}}\right)^{2}\left\{1-\frac{p_{0}}{p_{1}}+\left(\frac{p^{*}}{p_{1}}\right)^{4} \ln \left[\frac{p_{0}+p^{*}\left(p^{*} / p_{1}\right)^{2}}{p_{1}}\right]\right\} \text {. }
$$

Рассмотрим теперь область базового слоя, примыкающего к $n^{+}$-эмиттеру, в которой реализуется диффузионное приближение. Малый размер этой области $x_{2} \leq x \leq W$, именно $\left(W-x_{2}\right) / L<1$, позволяет линеаризовать решение уравнения (6) и представить его в виде

$$
p(x)=a_{1} x+a_{2},
$$

где

$$
\begin{gathered}
a_{1}=\frac{p_{W}-p_{2}}{W-x_{2}}, \quad a_{2}=-\frac{p_{W} x_{2}-p_{2} W}{W-x_{2}} \\
p_{W}=n_{i}\left[\frac{2}{(b+1)} \frac{j}{j_{i}}\right] /\left[1+\sqrt{1+\frac{4}{(b+1)} \frac{j j_{s p}}{j_{i}^{2}}}\right], \\
j_{i}=\frac{q D_{a} n_{i}}{W}, \quad p_{2}=p\left(x_{2}\right) .
\end{gathered}
$$

Концентрация носителей заряда $p_{2}$ в точке $x=x_{2}$ определяется условием (8). Учитывая, что из уравнения (11) следует условие $d p /\left.d x\right|_{x=x_{2}}=a_{1}$, выражение (8) можно переписать в виде

$$
\frac{j}{j_{d}}\left(\frac{j}{j_{c r}}-1\right) \frac{N_{d}^{2}}{p_{2}^{2} L} a_{1}=\frac{p_{2}}{L^{2}}
$$

Приближенное решение уравнения (12) имеет вид

$$
\frac{p_{2}}{p^{*}} \approx\left(\frac{p_{W} L}{p^{*} W}\right)^{1 / 3}
$$

Принимая во внимание сильную асимметрию инжектирующих способностей контактов Шоттки и $n-n^{+}$-перехода (условие $W / L<1$ ), можно определить положение точки $x_{2}$. С учетом соотношения (13) легко показать, что

$$
\frac{x_{2}}{L} \approx \frac{W}{L} \frac{p_{2}}{p_{W}}\left(1-\frac{p_{0}}{p_{2}}\right) \approx\left(\frac{p^{*}}{p_{2}}\right)^{2}\left(1-\frac{p_{0}}{p_{2}}\right) .
$$

Очевидно, что для последовательного описания вольт-амперной характеристики ДШ необходимо рассмотреть промежуточную область с координатами $x_{1} \leq x \leq x_{2}$. Концентрации носителей заряда $p$ и производная $d p / d x$ должны быть непрерывны в точках $x=x_{1}$ и $x=x_{2}$. Из соотношений (7) и (8) следует

$$
\begin{gathered}
\left.\frac{d p}{d x}\right|_{x=x_{1}}=\frac{p_{1}^{3}}{\left(j / j_{d}\right)\left(j / j_{c r}-1\right) N_{d}^{2} L}=\frac{p_{1}^{3}}{p^{* 2} L}, \\
\left.\frac{d p}{d x}\right|_{x=x_{2}}=a_{1}=\frac{p_{2}^{3}}{\left(j / j_{d}\right)\left(j / j_{c r}-1\right) N_{d}^{2} L}=\frac{p_{2}^{3}}{p^{* 2} L} .
\end{gathered}
$$


В области $x_{1} \leq x \leq x_{2}$ все члены уравнения непрерывности (2) одного порядка, а само уравнение непрерывности можно представить в виде

$$
\frac{d}{d x}\left(\frac{d p}{d x}-\frac{p^{* 2}}{L p}\right)=\frac{p}{L^{2}}
$$

Проинтегрируем уравнение (17) по области $x_{1} \leq x \leq x_{2}$, воспользовавшись выражениями (15) и (16). В результате получим

$$
\left(p_{2}-p_{1}\right)\left(\frac{p_{2}^{2}+p_{2} p_{1}+p_{1}^{2}}{p^{* 2}}+\frac{p^{* 2}}{p_{2} p_{1}}\right)=\frac{1}{L} \int_{x_{1}}^{x_{2}} p(x) d x .
$$

При вычислении интеграла в правой части уравнения (18) воспользуемся очевидной малостью величины $\left(x_{2}-x_{1}\right) / L$. Учтем также, что область $x_{1} \leq x \leq x_{2}$ содержит точку перегиба $x_{p}$. Тогда правую часть соотношения (18) можно записать в виде $\left(p_{2}+p_{1}\right)\left(x_{2}-x_{1}\right) / 2 L$. В результате ширина части с координатами $\left(x_{2}-x_{1}\right)$ может быть представлена в форме

$$
\frac{x_{2}-x_{1}}{L} \approx 2 \frac{p_{2}-p_{1}}{p_{2}+p_{1}}\left(\frac{p_{2}^{2}+p_{2} p_{1}+p_{1}^{2}}{p^{* 2}}+\frac{p^{* 2}}{p_{2} p_{1}}\right) .
$$

Соотношения (10), (14) и (19) позволяют представить связь между $p_{1}$ и $p_{2}$ в виде

$$
p_{1}=p_{2}\left(1-\frac{\delta p}{p_{2}}\right)
$$

где

$$
\frac{\delta p}{p_{2}}=\frac{1}{3}\left(\frac{p^{*}}{p_{2}}\right)^{8} \ln \frac{p_{2}}{p_{0}+p^{*}\left(p^{*} / p_{2}\right)^{2}} .
$$

Таким образом, выражение для ширины области $\left(x_{2}-x_{1}\right)$ имеет вид

$$
\frac{x_{2}-x_{1}}{L}=\left(\frac{p^{*}}{p_{2}}\right)^{6} \ln \left[\frac{p_{2}}{p_{0}+p^{*}\left(p^{*} / p_{2}\right)^{2}}\right] \text {. }
$$

Из малости отношения $p^{*} / p_{2}$ следует условие $p_{1} \approx p_{2}$, и отсюда неравенство $\left(x_{2}-x_{1}\right) \ll L$.

Полученный результат позволяет пренебречь областью $x_{1} \leq x \leq x_{2}$ и сшить решение (9) уравнения непрерывности (5) в области $0 \leq x \leq x_{1}$ и решение (11) уравнения непрерывности (6) в области $x_{2} \leq x \leq W$ в точке $x=x_{1}=x_{2}$. Полагая $p_{1}=p_{2}$ и воспользовавшись выражениями (15) и (16), получим

$$
\left.\frac{d p}{d x}\right|_{x=x_{2}-}=\left.\frac{d p}{d x}\right|_{x=x_{2}+}=\frac{p_{2}^{3}}{p^{* 2} L},
$$

где $p_{2}$ определяется выражением (13).
Найденные решения позволяют аналитически рассчитать вольт-амперную характеристику ДШ. Стандартное выражение для падения напряжения на ДШ имеет вид

$$
U=\frac{k T}{q} \ln \left(\frac{p(0) p(W)}{n_{i}^{2}}\right)+\int_{0}^{W} F(x) d x,
$$

где $p(0)$ и $p(W)$ - концентрации на границах базового слоя.

Для области $0 \leq x \leq x_{2}$ из уравнения (9) следует выражение для падения напряжения на этой области $U_{1}$ :

$$
U_{1}=\frac{2 b}{(b+1)^{2}} \frac{k T}{q} \frac{j W}{q D_{a} p_{W}} \ln \left(\frac{p_{2}}{p_{0}+p^{*}\left(p^{*} / p_{2}\right)^{3}}\right) .
$$

Падение напряжения $U_{2}$ на области $x_{2} \leq x \leq W$ легко рассчитать из уравнения (11):

$$
U_{2}=\frac{2 b}{(b+1)^{2}} \frac{k T}{q} \frac{j W}{q D_{a} p_{W}} \ln \left(\frac{p_{W}}{p_{2}}\right) .
$$

Полное падение напряжения $U_{W}$ на всей базовой области есть

$$
\begin{aligned}
U_{W}= & U_{1}+U_{2}=\frac{2 b}{(b+1)^{2}} \frac{k T}{q} \frac{j W}{q D_{a} p_{W}} \\
& \times \ln \left(\frac{p_{W}}{p_{0}+p^{*}\left(p^{*} / p_{2}\right)^{3}}\right) .
\end{aligned}
$$

Таким образом, с учетом выражения (24) вольт-амперная характеристика ДШ при $j>j_{c r}$ имеет вид

$$
\begin{aligned}
U= & \frac{k T}{q}\left\{\ln \frac{p_{0} p_{W}}{n_{i}^{2}}+\frac{2 b}{(b+1)^{2}} \frac{j W}{q D_{a} p_{W}}\right. \\
& \left.\times \ln \left[\frac{p_{W}}{p_{0}+p^{*}\left(p^{*} / p_{2}\right)^{3}}\right]\right\} .
\end{aligned}
$$

Полученное выражение отличается от выражения, описывающего вольт-амперную характеристику при $j<j_{c r}[14]$,

$$
\begin{aligned}
U= & \frac{k T}{q}\left\{\ln \frac{p_{0} p_{W}}{n_{i}^{2}}+\frac{2 b}{(b+1)^{2}} \frac{j W}{q D_{a} p_{W}}\right. \\
& \left.\times \ln \left[\frac{p_{W}}{p_{0}-p_{1}^{*}\left(p_{1}^{*} / p_{2}\right)^{3}}\right]\right\},
\end{aligned}
$$

где $p_{1}^{*}=N_{d} \sqrt{\left(j / j_{d}\right)\left(1-j / j_{c r}\right)}$.

Как нетрудно убедиться, из выражений (28) и (29) вытекает, что для обоих случаев остается справедливым следующее заключение: падение напряжения на ДШ растет с ростом уровня легирования базы $N_{d}$. Вместе c тем, соответствующее решение при использовании стандартного граничного условия предсказывает независимость падения напряжения от уровня легирования при высоком уровне инжекции $[7,16]$.

На рис. 2, $a$ показаны вольт-амперные характеристики кремниевых ДШ, рассчитанные в соответствии с 

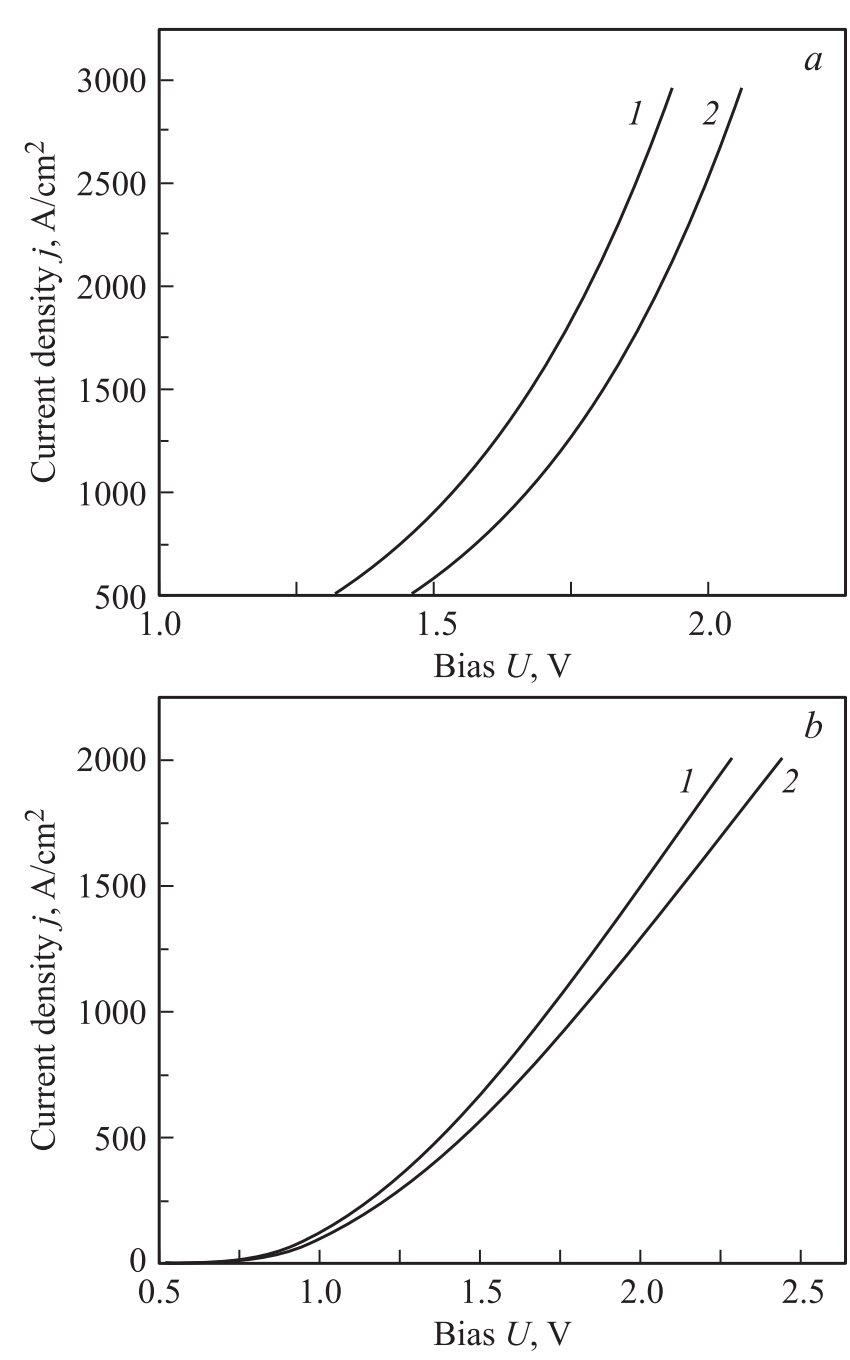

Рис. 2. Вольт-амперные характеристики диода Шоттки: $a$ - расчет в соответствии с выражениями (28), (29); $b-$ численный расчет с помощью программы INVESTIGATION (ISSLEDOVANIE). Уровень легирования базы $N_{d}, \mathrm{~cm}^{-3}$ : $1-5 \cdot 10^{13}, 2-5 \cdot 10^{14}$.

выражениями (28), (29) при следующих параметрах структур: толщина базового $n$-слоя $W=100$ мкм, отношение $W / L=0.5$; плотности токов насыщения контакта Шоттки $j_{s 0}$ и $n-n^{+}$-перехода $j_{s n}$ равнялись $2 \cdot 10^{-8}$ и $2 \cdot 10^{-12} \mathrm{~A} / \mathrm{cm}^{2}$ соответственно.

Расчет проводили для двух значений концентрации легирующей примеси в базовом слое: $N_{d}=5 \cdot 10^{13}$ и $5 \cdot 10^{14} \mathrm{~cm}^{-3}$. Как видно из рис. 2 , падение напряжения на диоде растет с ростом уровня легирования.

Результаты, полученные в рамках аналитической модели, были проверены с помощью численного эксперимента, осуществленного с помощью программы INVESTIGATION (ISSLEDOVANIE). Подробное описание этой программы приведено в работах $[17,18]$. Работа программы основана на численном решении уравнений непрерывности для электронов и дырок и уравнения Пуассона. В программе учтена полная совокупность нелинейных эффектов, обусловленных высоким уровнем инжекции в базовом слое и сильным легированием эмиттерной области.

Расчет проводили для кремниевого ДШ с толщиной базовой области $W=100$ мкм и толщиной $n^{+}$-эмиттера 20 мкм. Концентрация легирующей примеси в $n^{+}$-эмиттере составляла $1 \cdot 10^{19} \mathrm{~cm}^{-3}$. Время жизни носителей заряда в базовом слое при высоком уровне инжекции составило 22 мкс, что соответствовало отношению $W / L=0.5$. Высота барьера Шоттки для электронов $\varphi_{B n}=0.85$ эВ.

На рис. 2, $b$ представлены результаты расчета для двух значений концентрации легирующей примеси базового слоя: $N_{d}=5 \cdot 10^{13}$ и $5 \cdot 10^{14} \mathrm{~cm}^{-3}$. Поскольку при численных расчетах ограничения на величину плотности тока отсутствуют, расчет проведен начиная с относительно небольших плотностей тока.

Видно, что численные расчеты качественно полностью согласуются с выводами аналитической модели. С увеличением уровня легирования падение напряжения растет с ростом легирования базы. Этот вывод справедлив только при высоком уровне инжекции в базе ДШ. При низком уровне инжекции реализуется стандартная зависимость падения напряжения от уровня легирования: чем выше уровень легирования, тем меньше падение напряжения на ДШ при одинаковой плотности тока. В рассматриваемых структурах переход от низкого к высокому уровню инжекции соответствует плотности тока $j_{l h} \approx 20 \mathrm{~A} / \mathrm{cm}^{2}$. При плотности тока, соответствующей значению $j_{l h}$, кривые 1 и 2 на рис. $2, b$ пересекаются.

Сравнение данных, представленных на рис. 2, $a, b$ показывает, что имеется заметное различие в значениях падения напряжения $U$, полученных в рамках аналитических и численных расчетов. Анализ показывает, что основным источником неточности аналитического расчета является приближенный характер метода региональных приближений. Однако основной вывод аналитической теории остается справедливым во всех случаях.

\section{4. Заключение}

Аналитически исследован вид вольт-амперных характеристик диодов Шоттки в области больших плотностей тока в условиях, когда реализуется высокий уровень инжекции неосновных носителей заряда. При анализе использовано граничное условие для области базового слоя, примыкающей к контакту металл-полупроводник, учитывающее слабую инжекционную способность перехода метал-полупроводник. Показано, что „классическое“ граничное условие, обычно используемого для анализа работы диода Шоттки при высоком уровне инжекции, приводит к качественно неверному результату в области больших плотностей прямого тока. В соответствии с полученным аналитическим выражением, чем выше уровень легирования базы ДШ, тем больше 
падение напряжение на диоде при той же плотности тока в условиях высокого уровня инжекции неосновных носителей. Полученные результаты подтверждены численным расчетом в рамках модели INVESTIGATION, в которой учтена полная совокупность нелинейных эффектов, обусловленных высоким уровнем инжекции в базовом слое и сильным легированием эмиттерной области.

Работа выполнена при финансовой поддержке Российского фонда фундаментальных исследований (грант № 16-08-01038).

\section{Список литературы}

[1] L.M. Hillkirk. Solid State Electron., 48, 2181 (2004).

[2] M.E. Levinshtein, T.T. Mnatsakanov, P.A. Ivanov, J.W. Palmour, M.K. Das, B.A. Hull. Semicond. Sci. Technol., 23, 085011 (2008).

[3] J.W. Palmour, M.E. Levinshtein, P.A. Ivanov, Q.J. Zhang. J. Phys. D: Appl. Phys., 48, 235103 (2015).

[4] D.L. Scharfetter. Solid State Electron., 8, 299 (1965).

[5] A. Yu, E. Snow. Solid State Electron., 12, 155 (1969).

[6] B. Elfsten, P.A. Tove. Solid State Electron., 28, 721 (1985).

[7] W.T. Ng, S. Liang, C.A.T. Salama. Solid State Electron., 33, 39 (1990).

[8] K. Sarpatwari, S.E. Mohney, S. Ashok, O.O. Awadelkarim. Phys. Status Solidi A, 207, 1509 (2010).

[9] R. Singh, D.C. Capell, A.R. Hefner, J. Lai, J.W. Palmour. IEEE Trans. Electron Dev., 49, 2054 (2002).

[10] J.H. Zhao, K. Sheng, R.C. Lebron-Velilla. In: SiC Materials and Devices, vol. 1, ed. by M. Shur, S. Rumyantsev, M. Levinshtein (World Scientific, Singapore-New Jersey, 2006) p. 117.

[11] C. Buttay, C. Raynaud, H. Morel, G. Civrac, M.-L. Locatelli, F. Morel. IEEE Trans. Electron Dev., 59, 761 (2012).

[12] T.T. Mnatsakanov, M.E. Levinshtein, A.G. Tandoev, S.N. Yurkov. J. Appl. Phys., 105, 044506 (2009).

[13] T.T. Mnatsakanov, A.G. Tandoev, M.E. Levinshtein, S.N. Yurkov. Semicond. Sci. Technol., 24, 075006 (2009).

[14] T.T. Mnatsakanov, M.E. Levinshtein, A.G. Tandoev, S.N. Yurkov, J.W. Palmour. Solid State Electron., 121, 41 (2016).

[15] M.E. Levinshtein, T.T. Mnatsakanov, P.A. Ivanov, J.W. Palmour, M.K. Das, B.A. Hull. Solid State Electron., 52, 1802 (2008).

[16] M. Lampert, P. Mark. Current injection in solids, (N. Y.-London, Academic Press, 1970).

[17] T.T. Mnatsakanov, I.L. Rostovtsev, N.I. Philatov. Solid State Electron., 30, 579 (1987).

[18] T.T. Mnatsakanov. Phys. Status Solidi B, 143, 225 (1987).

Редактор Л.В. Шаронова

\section{Current-voltage characteristics of Schottky diodes at high current densities under minority carriers injection}

T.T. Mnatsakanov ${ }^{1}$, A.G. Tandoev ${ }^{1}$, M.E. Levinshtein, ${ }^{2}$, S.N. Yurkov' ${ }^{1}$, J.W. Palmour ${ }^{3}$

${ }^{1}$ All-Russia Electrotechnical Institute, 111250 Moscow, Russia

${ }^{2}$ loffe Institute, 194021 St. Petersburg, Russia

3 Wolfspeed, A Cree Company, 3026 East Cornwallis Rd., Research Triangle Park, NC 27709, USA

Abstract An analytical expression for a Schottky diode currentvoltage characteristic at high injection level of minority carriers has been obtained. It is shown that even at very high current densities, the higher the base doping level, the larger voltage drop across the diode. The physical mechanism responsible for this ,paradoxical“ result is analyzed. Analytical results are confirmed by simulation performed with software that takes into account the entire set of nonlinear effects, caused by high injection level and high doping level of the emitter region. 